\title{
Bloqueo del plano de la fascia clavipectoral guiada por ultrasonido
}

\author{
Ultrasound-guided clavipectoral fascia plane block
}

Hipólito Labandeyra MD. ${ }^{1}$, Vicente Roqués Escolar MD. ${ }^{2}$, Luis Fernando Valdés Vilches MD. ${ }^{3}$

\begin{abstract}
The case of a patient with clavicular middle third fractures is presented. The aim is explaining the ultrasound guided clavipectoral fascia plane block (CPB). This constitutes a novel technique and an alternative to traditional regional anesthesia of the brachial plexus. The purpose is to provide anesthesia, analgesia, and control over postoperative pain about the pathology mentioned. The technique was first described by L. Valdés in 2017.
\end{abstract}

\section{RESUMEN}

Se presenta el caso de una paciente con fractura de tercio medio clavicular con el objetivo de explicar el bloqueo del plano de la fascia clavipectoral guiado por ultrasonido (CPB). Ésta constituye una técnica novedosa y una alternativa a los procedimientos tradicionales de anestesia regional del plexo braquial, con el propósito de brindar anestesia, analgesia y control del dolor posoperatorio sobre dicha patología. Esta técnica fue descrita por primera vez en el año 2017 por L. Valdés[1].

\section{Key words:}

Clavicular middle third fracture,

clavipectoral fascia, postoperative pain, management, regional anesthesia

\section{Palabras clave:}

Fractura de tercio medio clavicular, fascia clavipectoral

Hospital Abraham Piñeyro, Junín. Buenos Aires, Argentina.

Hospital Virgen de Arrixaca. Murcia, España.

Hospital Costa del Sol. Marbella, España.

Fecha de ingreso: 09 de abril de 2020

Fecha de aceptación: 11 de noviembre de 2020

ORCID

0000-0002-1379-2516

Correspondencia:

Dr. Hipólito Landeyra

hipolitolabandeyra@gmail.com 


\section{Descripción del caso}

M ujer de 18 años con fractura de tercio medio clavicular derecho. Como antecedentes personales presentaba alergias a antiinflamatorios no esteroides y a contraste iodado. Previa realización del procedimiento se evaluó el dolor de la paciente mediante escala visual numérica (EVN) siendo de $7 / 10$ (Figura 1).

Se programa cirugía para osteosíntesis de clavícula mediante fijación con placa y tornillos. Como estrategia anestésica se decide realizar CPB asociado a infiltración del nervio supraclavicular (NSC) del plexo cervical superficial (PCS) y sedación.

Para el bloqueo NSC, con un transductor lineal de alta frecuencia (8-12 Mhz) se identificó la raíz C5 en el vértice del surco interescalénico. Luego se escaneó caudalmente hasta identificar el NSC como una estructura hipoecoica, de 1-3 mm de ancho, que se sitúa justo entre el borde superior del músculo escaleno medio y el borde posteroinferior del músculo esternocleidomastoideo, recordando que es el único nervio del PCS que viaja caudalmente. Se realiza un abordaje en plano de posterior hacia anterior y con una aguja fina de 35-50 $\mathrm{mm}$ de longitud se inyectan 2-3 $\mathrm{ml}$ de ropivacaína al 0,5\%[2] (Figura 2).

Para el bloqueo CPB se colocó la sonda sobre la clavícula, en plano parasagital y con orientación caudal, de manera que el haz de ultrasonidos incidió en forma exactamente perpendicular a la cara superior de la clavícula mientras que en la imagen de la pantalla ésta se visualizaba como una línea hiperecogénica paralela a la piel. A continuación, se realizó un barrido de toda la clavícula para identificar el trazo fracturario. Luego se desplazó el transductor hasta identificar: el periostio sano, la fascia clavipectoral e inmediatamente por encima de ésta y caudalmente el músculo pectoral mayor. A ambos lados de la fractura, con aguja de 35-50 mm, se realizó el abordaje en plano, desde caudal a cefálico hasta contactar con el periostio clavicular. Una vez contactado se retiró ligeramente la aguja infiltrando con ropivacaína 0,5\% observándose una dispersión caudocefálica de la solución anestésica y elevación de la fascia clavipectoral. El volumen total empleado fue de $30 \mathrm{ml}, 15 \mathrm{ml}$ a cada lado de la fractura inyectado sobre la cara superior de la clavícula[3],[4] (Figura 3).

La cirugía se llevó a cabo sin incidencias ni complicaciones, utilizando como sedoanalgesia $2 \mathrm{mg}$ de midazolam y 100 microgramos de fentanilo previo a la cirugía (Figura 4).

Realizada la conducta quirúrgica se evaluó el dolor del paciente con EVN, siendo de $1 / 10$ hasta $36 \mathrm{~h}$

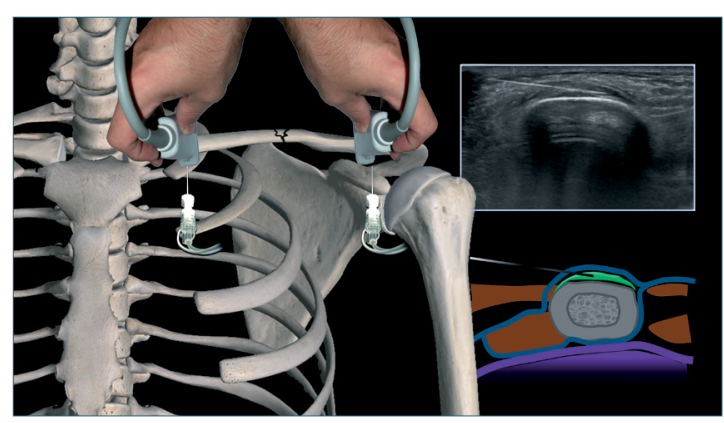

Figura 1. Rx de clavícula derecha.

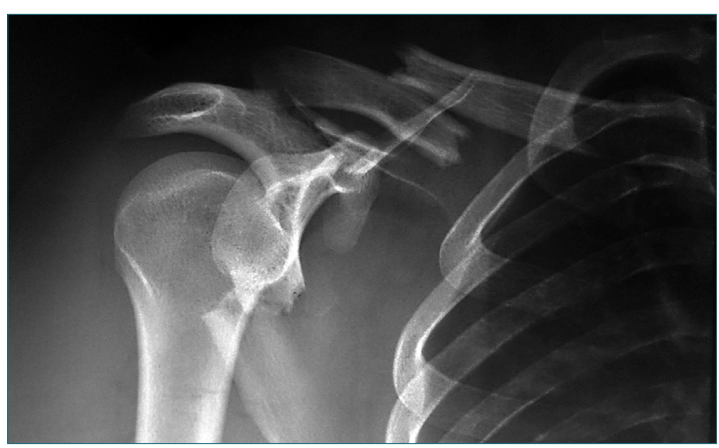

Figura 2. Esquema de la técnica de la punción de CPB e imagen ecográfica.

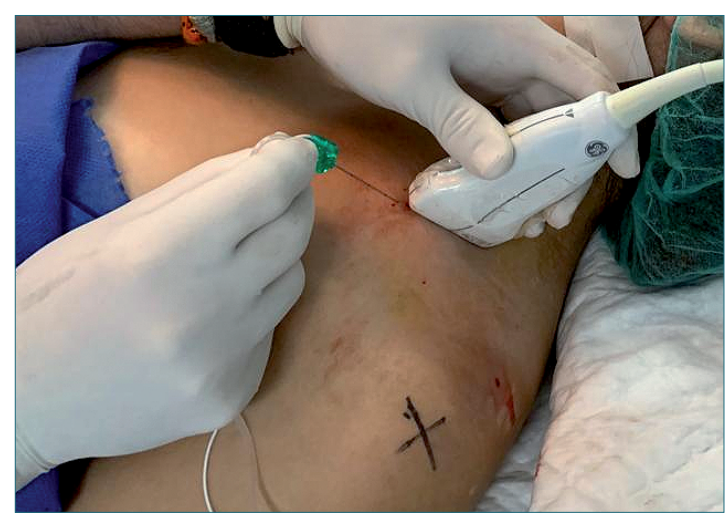

Figura 3. Técnica de punción. Posición del transductor y aguja.

posoperatorio. No fue necesario rescate analgésico ni se observó la presencia de bloqueo motor del miembro superior y tampoco parálisis diafragmática.

\section{Discusión}

Las fracturas de clavícula suponen el 2\%-3\% del 
total de fracturas y alrededor del $35 \%$ de la cintura escápula[5],[6]. Casi el 80\% de ellas acontecen a nivel del tercio medio clavicular, alrededor del $20 \%$ a nivel del tercio distal y solo una pequeña parte, $2 \%-3 \%$, a nivel del tercio proximal[6]. Se trata de una fractura con una intensidad de dolor postoperatorio moderado-severo[7] sobre el que, habitualmente, no se suele realizar ninguna técnica regional como componente analgésico mayor. Este hecho presuntamente se deba a la proximidad del plexo braquial a la fractura y al potencial riesgo de lesión nerviosa perioperatoria que conlleva esta cirugía. Ya está descrito que puede acontecer un déficit nervioso temporal de hasta en $7 \%-25 \%$ de las cirugías de clavícula[8]-[10].

Numerosas y complejas fascias se anclan a la clavícula con el fin de aportarle estabilidad y funcionalidad. La literatura ofrece diversas descripciones, que en algunos casos suelen ser contradictorias[11]. La mayor y más potente de todas es la fascia clavipectoral que precisamente es la que le da el nombre al bloqueo. Es una fascia compleja que se divide en varias hojas interrelacionándose con otras fascias. Se dispone en un plano coronal anclándose a nivel de los bordes anterior y posterior de la clavícula, envolviendo en su totalidad al músculo pectoral menor y caudalmente se inserta en los arcos costales $1^{\circ}$ a $5^{\circ}$. En su cara anterior establece una relación íntima con la fascia pectoral que envuelve al músculo pectoral mayor con la que se une y continúa cefálicamente por encima de la clavícula, fusionándose con las hojas de revestimiento y prevertebral de la fascia cervical profunda. En la cara inferior y borde posterior de la clavícula envuelve al músculo subclavio y una íntima relación con el ligamento coracoclavicular de Caldani. A nivel de la coracoides se extiende lateralmente hasta fusionarse con la fascia braquial para formar parte del ligamento suspensorio de la axila. De forma que, todas estas fascias crean una especie de envoltura circular sobre la clavícula. Los nervios que son responsables de la inervación sensorial de la clavícula llegan a la misma perforando dicha envoltura fascial. La inyección de una solución de anestésico local dentro de ésta permite una anestesia de la clavícula sin bloqueo de las ramas principales del plexobraquial[3].

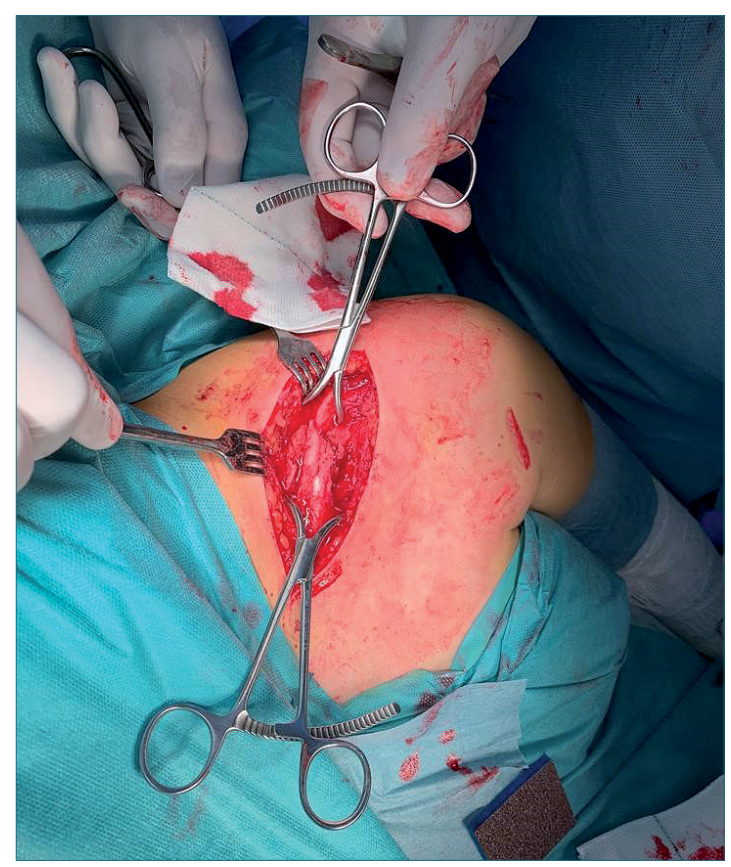

Figura 4. Técnica quirúrgica.

El bloqueo CPB para fracturas de tercio medio clavicular presenta varias ventajas respecto a las técnicas tradicionales de bloqueo del plexo braquial. Al constituir una técnica de simple ejecución, con referencias anatómicas concretas, estructuras superficiales y con un tope óseo para la aguja. No presenta bloqueo motor de los nervios principales del miembro superior al limitar la fascia clavipectoral la dispersión de anestésico local hacia el plexo braquial. No provoca parálisis diafragmática secundaria al bloqueo del nervio frénico lo que puede ser un provecho en ciertos casos, ya que este tipo de fracturas suelen acompañarse de lesiones costales que pueden generar disnea por dolor referido con agravamiento de la misma por la presencia de un bloqueo frénico.

Todo ello hace que el bloqueo CPB sea una técnica innovadora, efectiva y con reducidos riesgos potenciales.

\section{Referencias}

1. Valdés-Vilches L. (2017, September). Analgesia for clavicular surgery/fractures. In Symposia
01: Postoperative analgesia for Orthopedic upper and lower limb surgery. Symposium conducted at the 36th Annual European Society of Regional
Anaesthesia and Pain Therapy (ESRA) Congress, Lugano, Switzerland.

2. Maybin J, Townsley P, Bedforth $\mathrm{N}$, Allan A. Ultrasound guided 
supraclavicular nerve blockade: first technical description and the relevance for shoulder surgery under regional anaesthesia. Anaesthesia. 2011 Nov;66(11):1053-5. https://doi.org/10.1111/ j.1365-2044.2011.06907.x PMID:22004208

3. Ince I, Kilicaslan A, Roques V, Elsharkawy $\mathrm{H}$, Valdes L. Ultrasoundguided clavipectoral fascial plane block in a patient undergoing clavicular surgery. J Clin Anesth. 2019 Dec;58:125-7. https:// doi.org/10.1016/j.jclinane.2019.07.011 PMID:31377668

4. Roqués $V$, Valdés $L$, Pradere $A$, Elsharkawy H. Ultrasound guided CPB. https://www.youtube.com/ watch?v=gQ63x4ha7u8\&t=124s

5. Lenza M, Faloppa F. Surgical interventions for treating acute fractures or non-union of the middle third of the clavicle. Cochrane Database Syst Rev. 2015
May;(5):CD007428. https://
doi.org/10.1002/14651858.
CD007428.pub3
PMID:25950424

6. opars $\mathrm{M}$, Thomazeau $\mathrm{H}$, Huten D. Clavicle fractures. Orthop Traumatol Surg Res. 2017 Feb;103(1 1S):S53-9. https://doi. org/10.1016/j.otsr.2016.11.007 PMID:28043849

7. Herring $A A$, Stone $M B$, Frenkel O, Chipman A, Nagdev AD. The ultrasound-guided superficial cervical plexus block for anesthesia and analgesia in emergency care settings. Am J Emerg Med. 2012 Sep;30(7):1263-7. https://doi. org/10.1016/j.ajem.2011.06.023 PMID:22030184

8. Clitherow HD, Bain GI. Major neurovascular complications of clavicle fracture surgery. Shoulder Elbow. 2015 Jan;7(1):3-12. https://doi. rg/10.1177/1758573214546058

\section{PMID:27582950}

9. Robinson L, Persico F, Lorenz E, Seligson D. Clavicular caution: an anatomic study of neurovascular structures. Injury. 2014 Dec;45(12):1867-9. https://doi.org/10.1016/j. injury.2014.08.031 PMID:25306887

10. Wijdicks FJ, Van der Meijden OA, Millett PJ, Verleisdonk EJ, Houwert RM. Systematic review of the complications of plate fixation of clavicle fractures. Arch Orthop Trauma Surg. 2012 May;132(5):617-25. https://doi. org/10.1007/s00402-011-14565 PMID:22237694

11. Ince I, Kilicaslan $A$, Roques $V$, Elsharkawy $H$, Valdes L. The clavipectoral fascia plane block: reply to Dr. Altinpulluk. J Clin Anesth. 2020 May;61:109681. https://doi.org/10.1016/j. jclinane.2019.109681 PMID:31831283 